\title{
Omics-based profiling of systemic metabolic networks to decipher non-pharmacological mecha- nisms of action of natural complex substances
}

\author{
P. Sassone-Corsi \\ Center for Epigenetics and Metabolism, School of Medicine, University of California, Irvine, California
}

\section{Introduction}

The advent of the new EU Regulation 2017/745 should be welcomed as an exceptional opportunity to realize major advancements in the treatment of human diseases. Indeed, the introduction of a classification rule such as Rule 21 de facto acknowledges the existence and importance of medical devices composed of substances or their combination which, among others, require to be absorbed by the human body in order to achieve their efficacy. This innovative approach provides a much needed regulatory framework capable of defining the process to generate safe and efficacious therapeutic solutions, backed by credible clinical evidence and based on natural complex substances. The therapeutic potential of natural complex substances is known to mankind since millennia, yet, they are nowadays not regarded as source of therapeutic activities as much as they should. The potential compositional variability of natural complex substances and the intrinsically elusive nature of their mechanism of action, historically led to their decline in favor of the adoption of therapeutic solutions based on single molecules, often of synthetic nature, whose properties are very prone to be technically investigated via a reductionist approach. This, in turn, has led to the establish- ment of the key-lock (quali-quantitatively definable ligandreceptor binding event) model as the paradigm of election for the investigation of the mechanism of action of therapeutic activities.

Accordingly, the legislations concerning therapeutics have been designed to manage solutions based on single synthetic molecules, able to act via a key-lock mechanism, which in regulatory terms has been defined as the "pharmacological mechanism of action". This approach led to the disappearance of natural complex substances from the repertoire of credible therapeutic solutions available for treatment of human disease. The above described set of both technical and regulatory tools, the only one existing thus far, is in fact not applicable to the field of natural complex substances. Since such substances are mixtures of a great variety of chemical entities, in which both structural and functional interactions can occur, their properties (including PD/PK) are not definable as the algebraic sum of the properties of their single molecular components when studied in isolation (in search of a pharmacological mechanism of action), but are typical of the mixture per se. The properties of natural complex substances are therefore called "emerging properties", and quali-quantitative aspects of the mechanism of action underlying their establishment are, 
therefore, conceptually inaccessible through studies conducted with the reductionist paradigm of the "pharmacological mechanism of action" framework. It appears evident, then, that at least in regulatory terms the issue of describing the mechanism of action of a natural complex substance should hinge on the concept of "non-pharmacological", and a rather holistic approach to the generation of the data capable of describing it should be undertaken. EU Regulation 2017/745 provides the longawaited regulatory tool finally capable of considering peculiarities of natural complex substances within a "non-pharmacological" framework. Definitely the regulation provides for the establishment of requirements capable of rendering these substances a credible therapeutic solution. Yet, the set of techniques capable of approaching the issue of the depiction of the "non-pharmacological mechanism of action" of natural complex substances in a holistic fashion is currently left open. Recent technical advances in the field of omics- technologies, though, now enable researchers to decipher biological phenomena, therefore the consequences of the interaction of a substance with the human body, on a systemic, therefore holistic scale. Transcriptomics and metabolomics profiling of body fluids or organs paired to the use of ad hoc designed bioinformatics pipelines can return a comprehensive picture of the network of metabolic interactions occurring on a whole-body scale. Such tools can therefore acquire pivotal importance in the study of the mechanism of action of natural complex substances, which at that scale occurs.

The combination with other informatic approaches (e.g. chemoinformatics) capable of bridging features (among others, for instance, the chemical-physical properties and composition of the substance) to the biological effect exerted by a natural complex substance, will definitely be able to provide a satisfactory depiction of the mechanism of action of the natural complex substance. The following is a discussion of what application of the current state of the art can already enable to achieve.

\section{Circadian rhythms, Homeostasis and Holistic Biology}

Homeostasis, as initially described by Claude Bernard (1813-1878), identifies the capacity of higher animals, to maintain internal stability, owing to the coordinated physiological responses of its parts to any situation or stimulus that would tend to disturb its normal condition or function. Circadian (from the Latin "Circa" = around and "Diem" = day) rhythms constitute possibly the most exquisite system in the control of homeostasis. Circadian rhythms are controlled by molecular clocks- intrinsic, time-tracking systems that enable organisms to anticipate environmental changes (such as food availability and predatory pressure), allowing them to adapt their behavior and physiology to the appropriate time of the day. Indeed, the endogenous clock is synchronized to the daily 24-hour cycle generated by the rotation of our planet around its axis. It is worth noting that many fundamental biological processes function under circadian control underlining the relevance of circadian rhythms for whole body physiology and homeostasis. For instance, in humans, sleep/wake cycle, hormonal levels, blood pressure, metabolic reactions etc. are governed by specific endogenous clocks (Asher and Sassone-Corsi, 2015). The suprachiasmatic nucleus (SCN) in the hypothalamus is the master circadian timekeeper and is mainly entrained by light signals, transduced by specialized photoreceptors present in the retina. However, it has been demonstrated that different regions of the brain or peripheral organs, such as liver, intestine and heart also have local clocks, and that their proper function is necessary to regulate and preserve the physiology of every distinct tissue (Schibler and Sassone-Corsi, 2002). The role of the SCN is to keep these local clocks in synchrony, with each other and with the solar cycle, thereby ensuring that physiology across the entire organism is temporally integrated and thus maximally adaptive, although the underlying mechanisms are not well understood. At the cellular level, any biological process driven by endogenous cellular clocks results in oscillatory rhythms in gene expression, metabolism and behavior. The ability to anticipate daily fluctuations in the environment is a critical adaption across all living organisms. While light exposure is a dominant signal for synchronizing circadian clocks with the environment (so-called zeitgeber, from German, meaning time-giver), post-industrial technology has loosened restrictions on human activity that were previously imposed by the solar day. The resulting shift in sleep-wake cycles parallel alterations to the timing of physical activity and dietary intake, which are potent zeitgebers in their own right.

Disruptions in circadian rhythms resulting from our modern lifestyle are associated with familiar afflictions ranging from jet lag to mood and sleep disorders. The correlation of these cycles with human health and disease is well documented, and recent discoveries have re- 
inforced their biological importance. Unraveling the mechanisms driving circadian rhythms affirms their central role in physiology, and it has sparked further research to determine how external factors that interfere with our clocks might also influence the pathology of disease. Lifestyle risk factors such as diet and exercise are believed to play a role in the susceptibility of diseases such as diabetes, obesity, cardiovascular disease and cancer (Asher and Sassone-Corsi, 2015). Could the elevation or reduction in disease risk be mediated through our internal biological clocks?

\section{High-throughput Metabolomics reveal system-wide metabolic connections}

Understanding the physiological changes associated with oscillations of specific biomarkers is complex as it requires monitoring elaborate networks of metabolites across multiple tissues at various intervals of the circadian cycle. By applying high-throughput metabolomics in multiple mouse tissues we aimed at unraveling how naturally oscillating metabolic processes are integrated in a physiological network (Dyar et al. 2018). Our comprehensive circadian metabolomics resource provides temporal and spatial perspectives on circadian metabolite abundance, revealing the exquisite temporal intraand inter-tissue circadian communication that exists both under conditions of energy balance and energy imbalance. Importantly, we show how metabolites are linked within and across various tissues over time, and how they are modified by chronic nutrient stress in the form of chronic high fat diet feeding. By globally mapping relative metabolite distribution and abundance over time, in different tissues, and under different nutritional conditions, we have attempted to accurately capture the dynamic nature of tissue metabolism and inter-organ communication over 24 hours. Analyzing the number, amplitude, class, and peak distributions of circadian metabolites in each tissue, we further characterized tissues in terms of their circadian metabolism and defined precise temporal windows of common and tissue-specific metabolic pathways. Finally, our comparative analysis of metabolite correlations within and among tissues highlights coordinated metabolic pathways under physiological and pathological conditions (Dyar et al. 2018). This work leverages the study of metabolomics as a critical tool to better understand cellular physiology and to reveal connections between external inputs and pathology (Koronowski et al. 2019). The simultaneous evaluation of a comprehensive panel of multi-tissue metabolites - over multiple time intervals, and under varying environmental conditions allows a glimpse of the communication pathways between various organs that are essential to whole-organism homeostasis. In this case, the combined power of metabolomics with sophisticated analysis tools provides novel insight into the underpinnings of metabolic processes linked to phenotypic differences in mice, and it validates the use of this approach to support new discoveries in human health and disease.

\section{Temporal and tissue-specific metabolite signatures: the effects of nutrient stress}

Several studies from our team highlight the effect of nutritional abundance on circadian metabolism and demonstrate its relevance to the development and management of metabolic disease. Building on our previous work, we have used a systems biology approach to examine several tissues in the context of energy balance. By comparing the patterns of metabolism under a normal chow diet with conditions of nutrient stress imposed by HFD, they assembled a spatial and temporal atlas of circadian mouse metabolism. The atlas maps hundreds of circadian metabolites, revealing the metabolic connections that control daily oscillations in processes that are often mediated by distal organ systems. Furthermore, the study showed that external factors such as chronic nutrient stress can alter communication and coordination between tissue clocks, resulting in metabolic changes associated with pathology. Detection included a wide range of metabolite classes from 8 tissue types (i.e., serum, liver, skeletal muscle, brain, brown and white fat, and sperm). Alterations in the relative abundance of several metabolites were characteristic of known tissue-specific pathology. For example, carbohydrates comprised $53 \%$ of total altered liver metabolites of mice fed normal chow compared with only $8 \%$ in the HFD group. Lipid metabolites exhibited an inverse proportion, with $11 \%$ altered in mice on normal chow versus 52\% in HFD-fed mice. The accumulation of lipids in liver relative to carbohydrates is suggestive of HFD-induced hepatic steatosis and may have relevance to the progression of NASH (non-alcoholic steatohepatitis).

A similar shift in lipid accumulation in skeletal muscle, a prominent glucose sink, suggests the potential for de- 
velopment of insulin resistance (Dyar et al. 2018). Notably, several epidemiological studies have correlated an increased risk for insulin resistance and fatty liver with night shift work. To create a visual atlas of the metabolites under study, we have applied algorithms that plotted the significant temporal correlations according to metabolite class and tissue type. The resulting atlas revealed both temporal and tissue-specific signatures of metabolic pathways over the 24-hour cycle. When examining correlations according to metabolite class, serum lipids showed the greatest degree of synchronization with other metabolites under normal chow, consistent with a role for the vasculature in integrating biochemical networks. However, under HFD nutrient stress, these correlations were lost or significantly reduced, affirming the impact of energy balance on circadian misalignment.

\section{Potential applications in personalized medicine}

In addition to elucidating key spatial and temporal elements of energy metabolism, our studies provide a model for examining the relationship between other external factors and normal coherent networks across tissues. The proposed model is not limited to examining the effect of nutritional behavior on metabolic disease, or other behavioral interventions such as exercise.

Integrated analysis of the circadian metabolome, possibly by integrating observations from different omics platforms such as genomics, transcriptomics and microbiomics, offer potential for further discovery in disease pathways to reveal novel biomarkers and therapeutic targets, as well as fine-tuning clinical diagnostics. Diagnostic measures and drug dosing are typically scheduled irrespective of circadian metabolism.

Constraints of the clinician's timetable, ensuring appropriate time intervals between medication doses, or the requirements of sample collection (e.g., fasting plasma or morning urine) dictate scheduling rather than coordi- nating with biological clocks. Many commonly prescribed drugs work by targeting the products of circadian genes, and since their half-lives are often less than 6 hours, timing of administration might have a significant impact on their action or influence potential side effects. Metabolite comparisons across multiple tissues may provide insight that has been missing from studies of single biomarkers that represent only one tissue at a specific time point of the circadian metabolome (Dyar et al. 2018). While the relationship between coordination of peripheral clocks and pathology remains mostly unknown, the circadian atlas we have developed demonstrates how global metabolomics is essential to fill this information gap. Exploiting known oscillations might permit actionable insights including optimization of other external behaviors, improving the accuracy of diagnostics, and targeting specific time points to administer therapeutics. In the future, this same approach could be used on human samples to unlock biological discoveries hidden within the temporal dysregulation of metabolic processes and provide insights to develop personalized chronotherapy.

\section{References}

1. Schibler U, Sassone-Corsi P. A Web of Circadian Pacemakers. 2002; 111: 919-922.

2. Asher G, Sassone-Corsi, P. Time for food: the intimate interplay between nutrition, metabolism, and the circadian clock. 2015; 161: 84-92.

3. Dyar KA, Lutter D, Artati A, Ceglia N, Liu Y, Armenta D, Jastroch M, Schneider S, Mateo SD, Cervantes M, Abbondante S, Tognini P, Orozco-Solís R, Kinouchi K, Wang, C, Swerdloff RS, Nadeef S, Masri SM, Magistretti PJ, Orlando V, Borrelli E, Uhlenhaut NH, Baldi P, Adamski J, Tschöp MH, Eckel-Mahan $\mathrm{K}$, Sassone-Corsi P. Atlas of circadian metabolism reveals system-wide coordination and communication between clocks. 2018; 174: 1571-1585.

4. Koronowski KB, Kinouchi K, Welz PK, Zinna VM, Shi J, Sa$\operatorname{mad}$ M, Chen S, Smith JG, Magnan CN, Kinchen J, Li W, Baldi P, Benitah SA, Sassone-Corsi P. Defining the Independence of the Liver Circadian Clock. 2019; 177: 1448-1462. 